\title{
PTPRO-Associated Hepatic Stellate Cell Activation Plays a Critical Role in Liver Fibrosis
}

\author{
Xudong Zhang a Zhongming Tan ${ }^{\mathrm{a}}$ Youjing Wang ${ }^{\mathrm{a}}$ Junwei Tang ${ }^{\mathrm{a}}$ Runjiu Jiang ${ }^{\mathrm{a}}$ \\ Jiajie Hou ${ }^{a}$ Han Zhuo $^{a}$ Xiaochen Wang ${ }^{a}$ Jie Jia Xihu Qin ${ }^{b}$ Beicheng Sun ${ }^{a}$ \\ aLiver Transplantation Center of the First Affiliated Hospital, Nanjing Medical University, Nanjing,

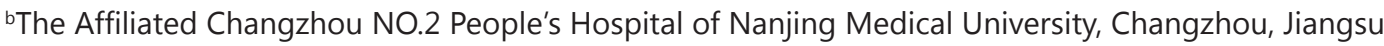 \\ Province, P.R. China
}

\section{Key Words}

PTPRO $•$ Liver fibrosis $•$ Bile duct ligation $\cdot \mathrm{CCl}_{4} \cdot$ Platelet-derived growth factor

\begin{abstract}
Background/Aims: PTPRO (protein tyrosine phosphatase, receptor type $O$ ) is implicated in diverse physiological and pathological processes in cancer and hepatic ischemia/ reperfusion injury, although little is known about its role in hepatic fibrosis. Methods: Here, by using genetically deficient mice, we reported that PTPRO knockout $\left(\mathrm{PTPRO}^{--}\right)$significantly attenuated liver injury, release of inflammatory factors, tissue remodeling, and liver fibrosis in two experimental mouse models of fibrogenesis induced by bile-duct ligation or carbon tetrachloride administration. Results: However, we proved that PTPRO expression was strongly downregulated in clinical and experimental liver fibrosis specimens. Further investigations revealed that stimulation of primary hepatic stellate cells (HSCs) and hepatocytes with specific activator platelet-derived growth factor (PDGF)-BB increased PTPRO transcription in HSCs but had the opposite effect in primary hepatocytes. More importantly, synthetic short hairpin RNA targeting PTPRO significantly neutralized PDGF-BB-induced HSC proliferation and myofibroblast marker expression through downregulated phosphorylation of extracellular signal-regulated kinase (ERK) and AKT. Conclusion: These observations confirm that PTPRO plays a critical role in liver fibrogenesis by affecting PDGF signaling in HSC activation and might be developed into a feasible therapeutic approach for the treatment of chronic fibrotic liver diseases.
\end{abstract}

X. Zhang and Z. Tan contributed equally to this work.

Beicheng Sun, M.D., Ph.D.,

or Xihu Qin, M.D., Ph.D.

\section{KARGER 125}

Liver Transplantation Center of the First Affiliated Hospital, Nanjing Medical University, 300 Guangzhou Road, 210029 Nanjing, Jiangsu Province (P.R. China) or The Affiliated Changzhou NO.2 People's Hospital of Nanjing Medical University, Changzhou, Jiangsu Province, (P.R. China) E-Mail sunbc@njmu.edu.cn or E-Mail qinxihu@126.com 


\section{Introduction}

Liver fibrosis is a pathological process arising from continuous wound-healing responses to liver injury, which encompasses infection-related hepatic fibrosis, metabolic-type fibrosis, and biliary-type fibrosis. As the injury proceeds, the extracellular matrix (ECM) such as collagen and fibronectin accumulates and forms a fibrous scar to nodules of regenerating hepatocytes, which predisposes to liver failure and hepatocellular carcinoma [1]. Hepatic stellate cells (HSCs) resident in the space between hepatocytes and liver sinusoidal endothelial cells (LSECs) are viewed as a predominant cellular source of ECM when activated [2, 3]. They normally store vitamin (retinoid) droplets in their cytoplasm, and are differentiated by injury-associated immunological processes into fibrogenic myofibroblasts that produce ECM and affect the function of hepatocytes and LSECs [4, 5]. Fibrosis progression and regression need specific and complicated signaling pathways, including chemokines and cytokines such as platelet-derived growth factor (PDGF), transforming growth factor (TGF)- $\beta$ and leptin [68]. Many recent studies have focused on the early events in liver fibrosis, such as immune interactions with interleukin (IL)-17 and IL-33 [9-11], but how they support fibrosis and activate HSCs requires verification.

Protein-tyrosine phosphatases (PTPs) can act in an opposite but equally important manner to protein-tyrosine kinases (PTKs), as a key part of post-translational modification strategies to regulate protein activities [12]. Protein tyrosine phosphatase receptor type 0 (PTPRO), also named glomerular epithelial protein 1 (GLEPP), is an important member of the receptor-type PTPs family that is expressed in humans, rats and rabbits. There are two types of PTPRO, full-length PTPRO (PTPRO-FL) expressed in kidney, brain, lung, liver and breast, and truncated PTPRO (PTPROt) which is predominantly expressed in B lymphocytes and macrophages [13]. PTPRO has been implicated in several biological processes, including neuronal growth and branching [14, 15], mammary epithelial cell morphogenesis [16], and as a biomarker in various types of carcinoma [17-20]. Previous research from our center has indicated that PTPRO expression promoted by estrogen receptor $\alpha$ contributes to the sex difference in incidence of hepatocellular carcinoma [21]. Furthermore, we also reported that PTPRO played a bidirectional role in hepatic ischemia/reperfusion (I/R) injury by affecting signal transducer and activator of transcription (STAT)3 activity [22]. However, the role of PTPRO in liver fibrosis is still unclear. Here, we identified PTPRO as a key mediator of hepatic fibrosis, and further explored the idea of therapeutic modulation of PTPRO in hepatic inflammation and fibrosis.

\section{Materials and Methods}

Patient samples

All human liver samples were collected at First Affiliated Hospital of Nanjing Medical University, China (Table 1). Informed consent was obtained from the patients before the specimens were obtained. Cirrhosis specimens $(n=18)$ were collected from patients undergoing liver transplantation for end-stage liver cirrhosis. Normal liver tissue $(n=8)$ was collected from patients receiving hepatoectomy for hepatic cavernous hemangioma. Liver samples were analyzed by quantitative real-time PCR, or fixed in $4 \%$ paraformaldehyde followed by paraffin embedding for immunoblotting.

Mouse models

Specific pathogen-free, male C57BL/6 mice, aged 8-10 weeks were obtained from the Experimental Animal Center of Nanjing Medical University. PTPRO knockout (KO) C57BL/6 mice were kindly donated by Dr. Bixby, University of Miami, USA and bred in the Model Animal Research Center of Nanjing University. Carbon tetrachloride $\left(\mathrm{CCl}_{4} ; 0.6 \mathrm{~g} / \mathrm{kg}\right)$ was administered intraperitoneally twice weekly for 4 weeks, mixed with corn oil (or oil for control), and the mice were sacrificed 3 days after the final dose, when transaminase levels should return to near normal, as described previously [23]. For bile duct ligation (BDL), mice were anesthetized with 1\% pentobarbital. After midline laparotomy, the common bile duct was dissociated and ligated twice with 6-0 silk sutures, and the abdomen was closed. Sham operation was performed similarly, 
Table 1. Clinical characteristics. Data are presented as mean (minimum-maximum). ALT, alanine aminotransferase; HBV, hepatitis B virus (Others are alcoholic cirrhosis)

\begin{tabular}{lll}
\hline Characteristics & Fibrotic Liver & Control Liver \\
\hline $\mathrm{N}$ & 18 & 8 \\
$\mathrm{Sex}$ & 20 male & 7 male \\
Mean age (minimum-maximum), $\mathrm{y}$ & $52.2(40-59)$ & $44.1(32-55)$ \\
$\mathrm{HBV}$ infected, $\mathrm{n}$ & 16 & 3 \\
Mean ALT level (minimum-maximum), IU/l & $50.8(32.5-68.3)$ & $29.6(13.6-43.3)$ \\
Mean total bilirubin level (minimum-maximum) & $15.6(4.2-34.1)$ & $10.1(5.1-17.6)$ \\
Mean serum albumin (minimum-maximum), g/l & $32.5(25-42)$ & $36.2(32-45)$ \\
Complicated with hepatocellular carcinoma, $\mathrm{n}$ & 9 & 0 \\
\hline
\end{tabular}

except that the bile duct was not ligated [24]. To eliminate the influence of other complications, animals were sacrificed 3 or 14 days after BDL. Serum and liver were prepared for other research. Indicated groups of BDL challenged mice were observed until 21 days for overall survival rate analysis,

Microscopic investigation of liver after BDL or CCI4 treatment

Fragments of liver tissues were fixed in $4 \%$ buffered formaldehyde. Sections ( $3 \mu \mathrm{m})$ of liver were cut and stained with hematoxylin and eosin (H\&E). Liver H\&E sections were analyzed for I/R injury under a Leica DM4000 B Upright Research Microscope (100×).

Measurement of plasma alanine aminotransferase (ALT) activity

Blood was obtained by cardiac puncture at the time of sacrifice for analysis of serum ALT as an index of hepatocellular injury. Measurements of serum ALT were made using a diagnostic kit (Sigma, St. Louis, MO, USA).

HSCs and hepatocytes isolation and restimulation assay

Primary mouse HSCs were isolated from livers of C57BL/6 mice, as described previously, with modification [10]. Livers were perfused in situ with $45 \mathrm{ml}$ Gibco Liver Perfusion Media (Invitrogen, Carlsbad, CA, USA) followed by $45 \mathrm{ml}$ Gibco Liver Digestion Media (Invitrogen). The liver digests were filtered through a cell strainer and washed with Gey's Balanced Salt Solution (GBSS) (Sigma) containing DNase I (2 mg/ml, Roche Diagnostics). The homogenate was centrifuged at $25 \mathrm{~g}$ for $5 \mathrm{~min}$ at room temperature to remove the hepatocytes. The supernatant was transferred to a new tube and centrifuged at $400 \mathrm{~g}$ for $10 \mathrm{~min}$ at $4^{\circ} \mathrm{C}$. The cell pellet was resuspended in $5 \mathrm{ml}$ 15\% OptiPrep (Sigma), and loaded carefully with $5 \mathrm{ml}$ of 11.5\% OptiPrep and $2 \mathrm{ml} \mathrm{GBSS}$. After centrifugation at $1400 \mathrm{~g}$ for $17 \mathrm{~min}$ at $4^{\circ} \mathrm{C}$, the cell fraction in the GBSS and $11.5 \%$ OptiPrep interphase was gently aspirated, mixed with GBSS, and centrifuged at $1400 \mathrm{~g}$ for $10 \mathrm{~min}$ at $4^{\circ} \mathrm{C}$. After another wash, the final cell pellet was resuspended in Dulbecco's Modified Eagle's Medium (DMEM) containing penicillin, streptomycin and 10\% fetal bovine serum (FBS) (complete medium), and then plated on uncoated plastic at a density of $5 \times 10^{6}$ per 10 -cm diameter plate. After the first $24 \mathrm{~h}$, nonadherent cells and debris were removed by washing. Cell viability was $>90 \%$ as assessed by Trypan blue exclusion. Purity was 90-95\% as assessed by light microscopy. Primary murine hepatocytes were isolated from male C57BL/6 mice aged 6-8 weeks, as previously reported [22]. Isolated HSCs and hepatocytes were placed into the 96 well plate $\left(8 \times 10^{4}\right.$ cells/well) in DMEM and exposed to recombinant (rm) PDGF-BB (Peprotech, UK) (5ng/ $\mathrm{ml}$ [ [25] . After $12 \mathrm{~h}$ incubation at $37^{\circ} \mathrm{C}$, cells were harvested for other tests.

\section{RNA interference analysis}

PTPRO silencing by mouse PTPRO small hairpin RNA (shRNA) (5'-GCG CTC ATA CCG AAT GTC AAT CTC GAG ATT GAC ATT CGG TAT GAGCGC-3') or scrambled sequences (5'-TGACAGTCGATTGATGTGATT-3') designed and synthesized by Wuhan Genesial Corporation (Wuhan, China) was tested in HSCs. PTPRO levels following shRNA silencing were analyzed by RT-PCR and immunoblotting using anti-PTPRO antibodies. Such analysis revealed that the PTPRO-shRNA silenced PTPRO expression by $\sim 90 \%$ at a relatively low concentration (50 nmol/l) using Lipofectamine 2000 (Invitrogen) for three independent transfection experiments.

\section{KARGER}


Table 2. Primer used

\begin{tabular}{lll}
\hline Genes & Forward $\left(5^{\prime}-3^{\prime}\right)$ & Reverse $\left(5^{\prime}-3^{\prime}\right)$ \\
\hline PTPRO(Mouse) & ATTGGACTGGATATTCCGCACT & CAGGATGTTTGTGTAGCGGTTT \\
PTPRO(Human) & ATGACTTCAGCCGTGTGAGAT & GGGTGGCAATATACTCCTGGG \\
$\alpha-$ SMA & GTCCCAGACATCAGGGAGTAA & TCGGATACTTCAGCGTCAGGA \\
Col-1 $\alpha 1$ & TTCACCTACAGCACGCTTGTG & GATGACTGTCTTGCCCCAAGTT \\
MMP-2 & CAAGTTCCCCGGCGATGTC & TTCTGGTCAAGGTCACCTGTC \\
TIMP-1 & GCAACTCGGACCTGGTCATAA & CGGCCCGTGATGAGAAACT \\
TGF- $\beta$ & CTCCCGTGGCTTCTAGTGC & GCCTTAGTTTGGACAGGATCTG \\
GAPDH(Mouse) & AGGTCGGTGTGAACGGATTTG & TGTAGACCATGTAGTTGAGGTCA \\
GAPDH(Human) & ACAACTTTGGTATCGTGGAAGG & GCCATCACGCCACAGTTTC \\
\hline
\end{tabular}

\section{Real-time quantitative PCR}

RNA was isolated from mouse or human liver tissue by homogenization and purification using TRIzol reagent (Invitrogen). RNA was isolated from HSCs using RNeasy. Following DNase treatment and reverse transcription, real-time quantitative PCR of mouse and human samples was performed for 40 cycles of 15 $\mathrm{s}$ at $95^{\circ} \mathrm{C}$ and $1 \mathrm{~min}$ at $60^{\circ} \mathrm{C}$ using a one-step RT-PCR kit (TaKaRa, Dalian, China). The specific primers used are summarized in Table 2. Quantification was performed by comparing the Ct values of each sample with a standard curve and normalization to GAPDH. Values were expressed as fold induction in comparison with untreated or sham controls.

\section{Immunohistochemistry}

Immunohistochemistry of $\alpha$-smooth muscle actin (SMA) was performed using 5 - $\mu$ m paraffin sections. The sections were deparaffined in xylene and rehydrated in alcohol and distilled water. Antigen retrieval was performed by heating sections in solution (10 mM citrate buffer, pH 6.0) in a pressure cooker. $\mathrm{H}_{2} \mathrm{O}_{2}$ (3\%) was used to eliminate endogenous peroxidase. Slides were washed with Tris-buffered saline (TBS)Triton three times and once with TBS for 5 min. Nonspecific binding was blocked using 5\% goat serum for $30 \mathrm{~min}$. The blocking buffer was removed and sections were incubated with a polyclonal $\alpha$-SMA antibody (AB5694; Abcam, Cambridge, UK). Control experiments were incubated with rabbit serum replacing the first antibody. After incubation overnight at $4^{\circ} \mathrm{C}$ and washing, the sections were incubated with biotinylated goat anti-rabbit IgG antibody. After washing, peroxidase-coupled antibody was applied for 30 min at room temperature. Bound antibody was detected with 3, 3'-diaminobenzidine tetrachloride (DAB) (SigmaAldrich, St. Louis, MO, USA). All sections were counterstained with hematoxylin. Image-ProPlus version 6.0 was used to evaluate the areas of positive $\alpha$-SMA as computer-assisted semi-quantitative.

\section{Collagen detection assay}

Supernatants of liver homogenate samples were collected from mice; cell medium were obtained from $5 \mathrm{ng} / \mathrm{ml} \mathrm{rmPDGF-BB}$ restimulation for $12 \mathrm{~h}\left(1 \times 10^{5} \mathrm{cells} / \mathrm{ml}\right.$ in 6 -well plates). The collagen concentration was measured using the Sircol assay according to the manufacturer's recommended procedure (S1000, Biocolor, Carrickfergus, UK). Masson's trichrome stain and hydroxyproline assay were used to focus on the change in hepatic collagen content in vitro and in vivo. The detailed methods were according to the manufacturer's recommended procedure (HT15; Sigma-Aldrich and KGT030-2; KeyGEN Biotech, Nanjing, China).

\section{Cytotoxicity and viability assays}

Cell viability was assessed using the MTT reduction assay. Formation of blue formazan was measured spectrophotometrically from the metabolism of MTT by mitochondrial dehydrogenases that are active only in live cells. Isolated HSCs were seeded in 96 -well plates $\left(1 \times 10^{4}\right.$ cells per well) and then incubated in DMEM containing penicillin, streptomycin and 10\% FBS for $24 \mathrm{~h}$. The cells were pretreated with various concentrations of rmPDGF-BB for $12 \mathrm{~h}$, and MTT ( $5 \mathrm{mg} / \mathrm{ml})$ was added to each of the wells, and the plate was incubated for an additional $1 \mathrm{~h}$ at $37^{\circ} \mathrm{C}$. The medium was removed, and the intracellular formazan product was dissolved in $250 \mu \mathrm{l}$ DMSO. The absorbance of each well was measured at $540 \mathrm{~nm}$ using a microplate reader. OD values from untreated control cells were designated as $100 \%$ viability. Triplicate specimens were 


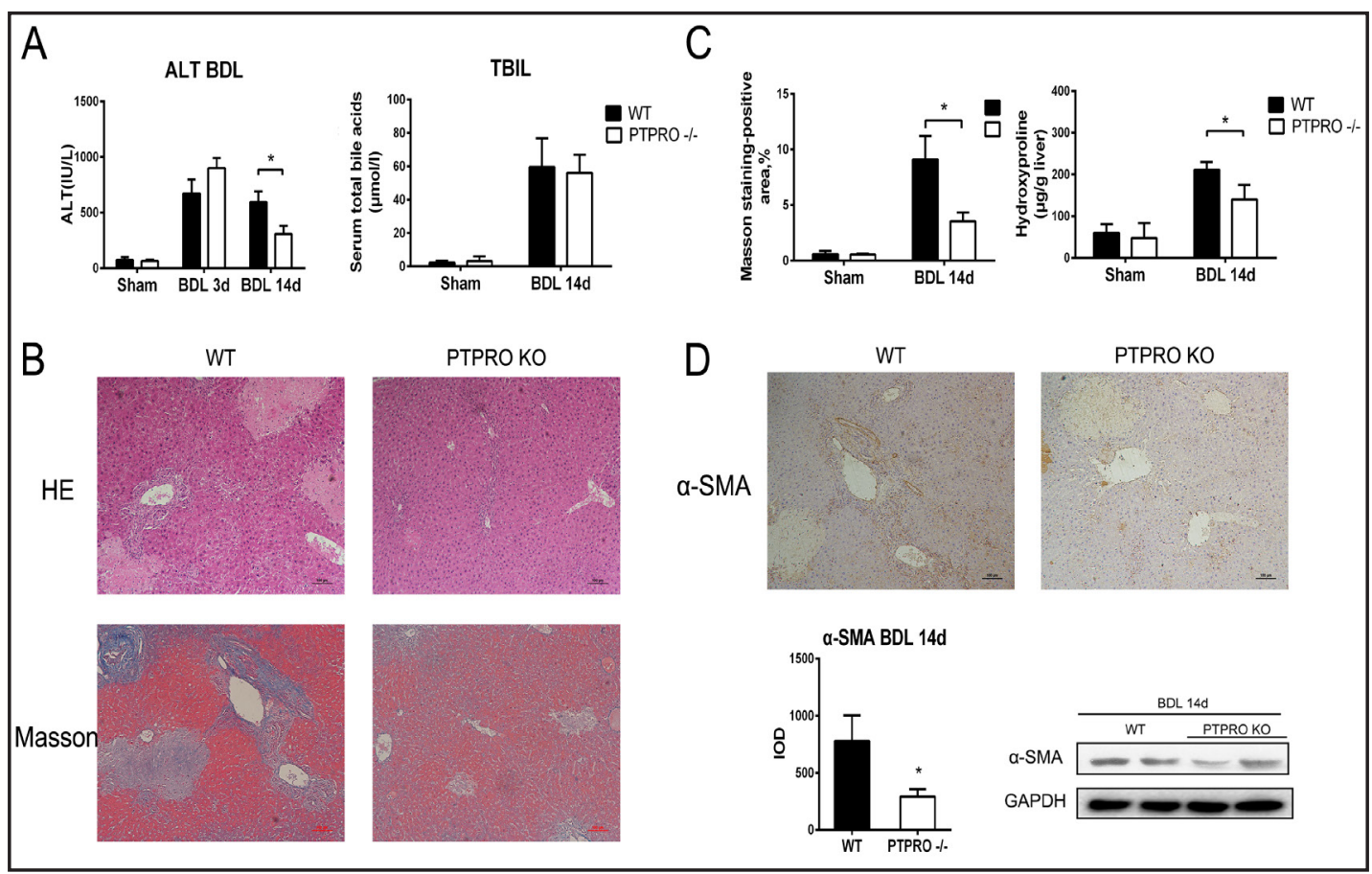

Fig. 1. PTPRO is required for hepatic fibrogenesis in BDL. Mice underwent BDL and were sacrificed 3 or 14 days later. (A) Serum ALT values and total bilirubin (TBil) were measured 3 and 14 days after BDL from WT and PTPRO ${ }^{--}$mice ( $\mathrm{n}=8$ per group) compared to each sham-operated control $(\mathrm{n}=4)$. TBil in BDL mice was almost identical, indicating a similar cholestatic effect of BDL between the groups of animals. (B) H\&E showed that the WT group had more widespread bile infarctions and cholestatic hepatitis than the PTPRO ${ }^{-/-}$ group had. Fibrillar collagen was determined by Masson staining (Collagen fibers stained as blue and cytoplasm stained as red, original magnification, 100×). (C) Quantification of positive area by Masson staining (left). Hydroxyproline quantification indicated that collengen deposition was increased in BDL-treated liver (right). (D) Expression of $\alpha$-SMA was measured by immunohistochemistry. Western blotting of $\alpha$-SMA between the two groups. Average integrated optical density (IOD) was obtained by analyzing five fields for each slide evaluated by Image-Pro Plus software (version 5.0) for immunohistochemical staining $\left({ }^{*} P<0.05\right)$.

repeated three times independently. Comparisons between groups were performed by one-way ANOVA followed by the Newman-Keuls post test.

\section{Western blotting}

Total cell (or tissue) protein was extracted on ice by homogenization and the concentration was determined using the Bradford method with a Bio-Rad protein assay, Protein extract (20 mg/lane) was separated by SDS-PAGE and transferred to PVDF membranes (Millipore, Billerica, MA, USA). After the blockade of nonspecific protein binding with $5 \%$ milk or serum, the membrane was incubated at a dilution with different antibodies. Immunoblotting was performed using antibodies against PTPRO (\#12161; Proteintech Group, Chicago, IL), $\alpha$-SMA, rabbit monoclonal antibodies against ERK1/2 (\#9102), phosphoERK1/2 (\#9101s), AKT (\#4691), and phospho-AKT (T308) (\#9275) (Cell Signaling Technology, Danvers, MA, USA) at a dilution of 1:1000. Blots were reprobed with mouse GAPDH antibody (Cell Signaling Technology). Horseradish-peroxidase-linked anti-rabbit and anti-goat antibodies were used as secondary antibodies. The results were exposed to autoradiography film (Kodak XAR Film) after the enhanced chemiluminescence light method.

\section{Statistical analysis}

All data was expressed as mean \pm SEM. Student $t$ tests were used to calculate statistical significance for data sets following normal distribution. Log-rank test were used to analyze the survival rate. $P<0.05$ was considered statistically significant. Some figures were prepared by GraphPad Prism 6. 


\section{Results}

\section{BDL-induced chronic inflammation and liver fibrosis is PTPRO dependent}

To test the functional importance of PTPRO in hepatic fibrosis, paired PTPRO KO mice and littermate WT mice were used in two kinds of models as mentioned above. In the model of BDL, total bilirubin (TBil) in challenged mice was measured, indicating a similar cholestatic effect of BDL between WT and PTPRO KO mice. The level of alanine aspartate aminotransferase in serum of mice confirmed the liver injury induced by BDL. ALT activity was reduced in PTPRO KO mice compared with WT mice at 14 days but there was no significant difference at 3 days (Fig. 1A). H\&E staining showed that mouse liver at 14 days after BDL demonstrated severe cholestatic hepatitis with widespread bile infarctions, bile ductular proliferation, and hepatocellular damage, whereas in $\mathrm{PTPRO}^{-/-}$mice there was less biliary injury (Fig. 1B). Furthermore, Masson's trichrome staining and hydroxyproline quantification indicated significant reduction of collagen deposition in $\mathrm{PTPRO}^{-/-}$mice (Fig. 1B, 1C). Moreover, $\alpha$-SMA, a cardinal marker for HSC activation, was increased in BDLchallenged mice as compared to sham-operated mice. By using immunohistochemistry and western blotting, we showed that $\alpha$-SMA immunoreactivity was increased in the sinusoids of BDL WT mice but reduced in BDL PTPRO ${ }^{-/-}$mice (Fig. 1D).

\section{PTPRO deficiency protects against CCl4-induced chronic hepatic injury and fibrosis}

PTPRO deficiency largely protected mice from BDL-associated liver injury and fibrosis, therefore, another model was introduced to confirm the role of PTPRO in chemically induced liver fibrosis. $\mathrm{CCl}_{4}$ was injected into $\mathrm{C} 57 \mathrm{BL} / 6$ or PTPRO $\mathrm{KO}$ mice at $0.6 \mathrm{~g} / \mathrm{kg}$ twice weekly. After 4 weeks, mice were sacrificed. The severity of liver injury in the PTPRO KO group was
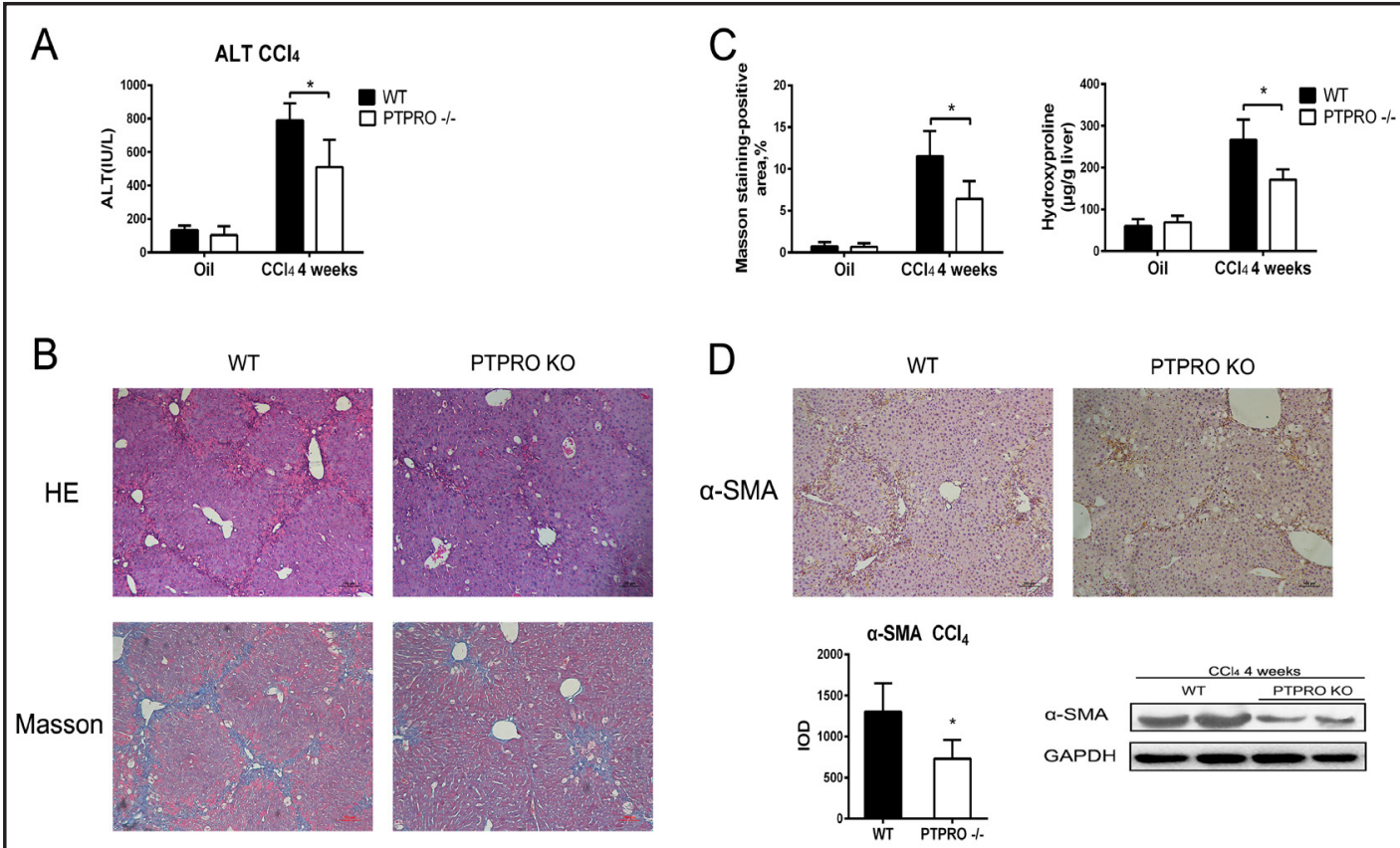

Fig. 2. PTPRO is required for hepatic fibrogenesis in $\mathrm{CCl}_{4}$ treatment. $\mathrm{C} 57 \mathrm{BL} / 6$ and $\mathrm{PTPRO} /$ - mice were administered intraperitoneally $0.6 \mathrm{~g} / \mathrm{kg} \mathrm{CCl}_{4}$ (diluted in corn oil) or oil alone twice weekly for 4 weeks, and mice were sacrificed at $48 \mathrm{~h}$ after the final challenge. (A) Serum ALT values were measured from WT and PTPRO $^{-/-}$mice. (B) Representative H\&E (original magnification, $100 \times$ ) and collagen deposition in liver sections (Masson trichrome, original magnification, $100 \times$ ) from C57BL/6 and PTPRO ${ }^{-/-}$mice injected with $\mathrm{CCl}_{4}$ or oil are shown. (C) Quantification of positive area of IOD by Masson staining and quantification of hydroxyproline content. (D) Expression of $\alpha$-SMA was measured by immunohistochemistry while IOD in D was obtained, and expression of $\alpha$-SMA was measured by western blotting. 


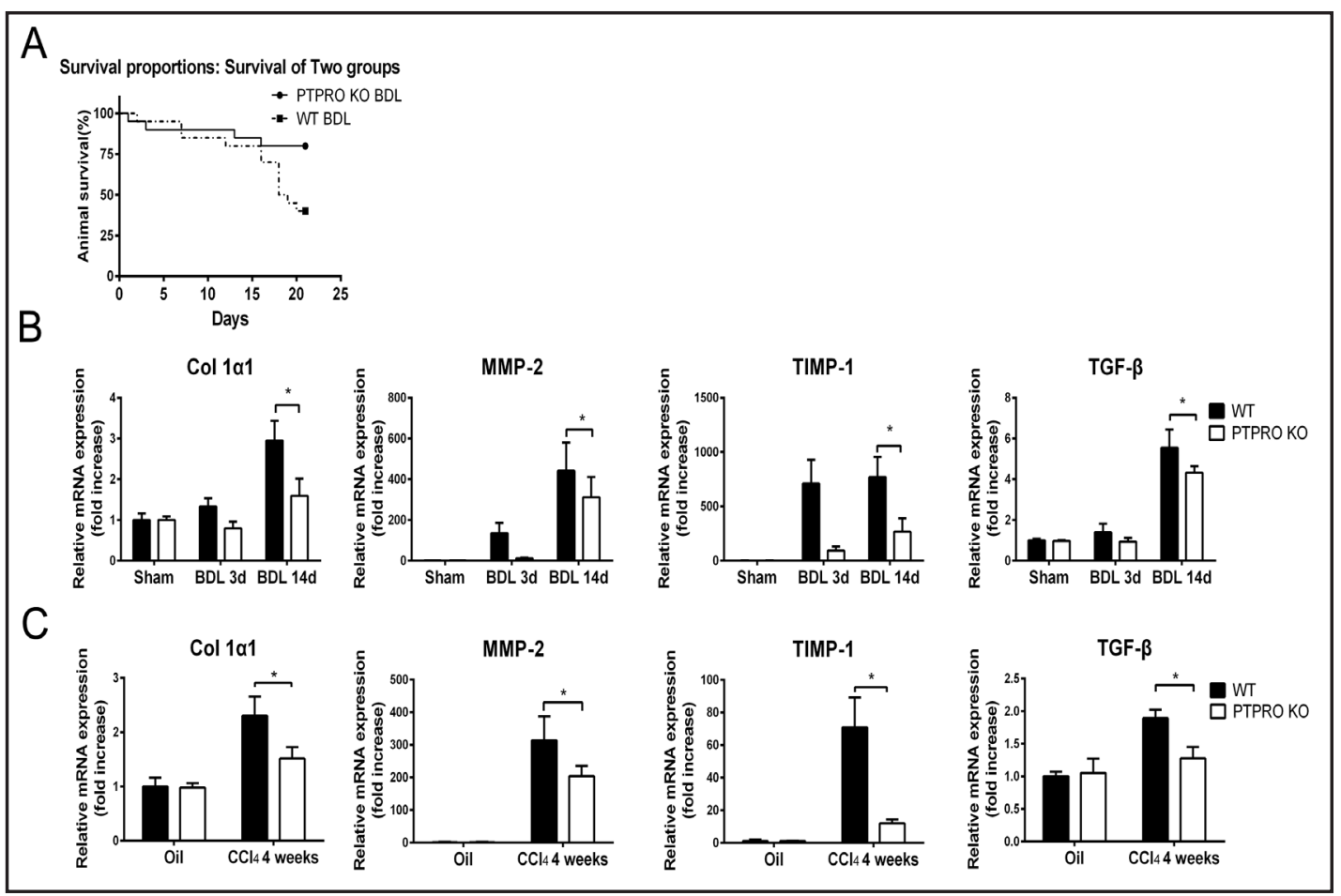

Fig. 3. Inflammatory cytokine expression and animal survival following liver fibrosis are dependent upon PTPRO. (A) Animal survival following BDL was enhanced in PTPRO ${ }^{-/-}$mice $(n=20)$. Initially on day 14 after BDL, $85 \%$ of $\mathrm{PTPRO}^{-/-}$mice were alive, whereas $80 \%$ of the WT animals survived cholestatic liver injury and fibrosis. However on day 21 after BDL, $80 \%$ of PTPRO $^{-/}$mice were still alive compared to only $40 \%$ in the WT group (log-rank test, $P=0.04,95 \% \mathrm{CI}=1.206-8.588$ ). (B) Expression of profibrogenic markers Col-1 $\alpha 1$, MMP-2, TIMP-1 and TGF- $\beta$ was determined by quantitative real-time PCR in BDL or sham-operated mice, which indicated that BDL-induced liver inflammation and fibrosis are PTPRO dependent. (C) Expression of those factors as mentioned above in $\mathrm{CCl}_{4}$-treated mice $\left({ }^{*} P<0.05\right)$.

relatively milder, by analyzing serum ALT level (Fig. 2A). Both Masson's and H\&E staining of the liver sections showed that 4 weeks repeated $\mathrm{CCl}_{4}$ injection resulted in obvious vesicular degeneration and uniform fibrous tissue deposition in WT mice, which was attenuated in PTPRO KO mice (Fig. 2B). As for the quantitative grading, the Masson staining-positive area and hepatic hydroxyproline content of the $\mathrm{PTPRO}^{-/-}$mice were significantly lower than in the WT group (Fig. 2C). This suggested that PTPRO-deficient mice showed reduced accumulation of collagen and had a protective role in $\mathrm{CCl}_{4}$-induced hepatic fibrosis. These findings were confirmed by the reduction in Immunocytochemical staining and western blotting for $\alpha$-SMA (Fig. 2D).

\section{Inflammatory cytokine expression and animal survival levels following liver fibrosis are dependent upon PTPRO}

Although PTPRO deficiency largely protected the mice from BDL- and $\mathrm{CCl}_{4}$-induced chronic liver injury and liver fibrosis, the exact mechanisms underlying this protective capacity remains unclear. Therefore, we investigated the inflammatory cytokine expression profiles in C57BL/6 and PTPRO ${ }^{-/-}$mice challenged in these two models. We first examined the overall survival in BDL-challenged mice. By day 21 after BDL, PTPRO-/- mice $(n=20)$ exhibited longer overall survival than the WT group (n=20) ( $80 \%$ vs $40 \%$, respectively, logrank test $P=0.04,95 \% \mathrm{CI}=1.206-8.588$ ) (Fig. 3A). Considering that the PTPRO deletion is not liver specific, the organ-specific effects responsible for enhanced animal survival could not be specifically delineated. However, because PTPRO KO reduces liver injury, hepatoprotection from BDL likely and partly contributes to the improved survival. 
Fig. 4. PTPRO downregulated in murine and human liver fibrosis. Fibrotic and normal liver tissues were collected from patients undergoing partial hepatectomy for early-stage hepatocellular carcinoma or hepatic hemangioma. (A) PTPRO mRNA transcript level was downregulated more in human fibrotic liver $(n=18)$ than normal liver $(\mathrm{n}=8)$. BDL $(\mathrm{n}=8)$ and $\mathrm{CCl}_{4}$ injection $(n=8)$ were performed to induce liver fibrosis in WT mice. (B and C) Expression of PTPRO and $\alpha$-SMA in mouse liver was tested by western blotting or quantitative PCR, and the results were shown as fold changes compared with each control $(n=4)$ (Data were from independent samples and expressed as mean $\pm \mathrm{SEM},{ }^{*} P<0.05$ by Student $t$ tests).

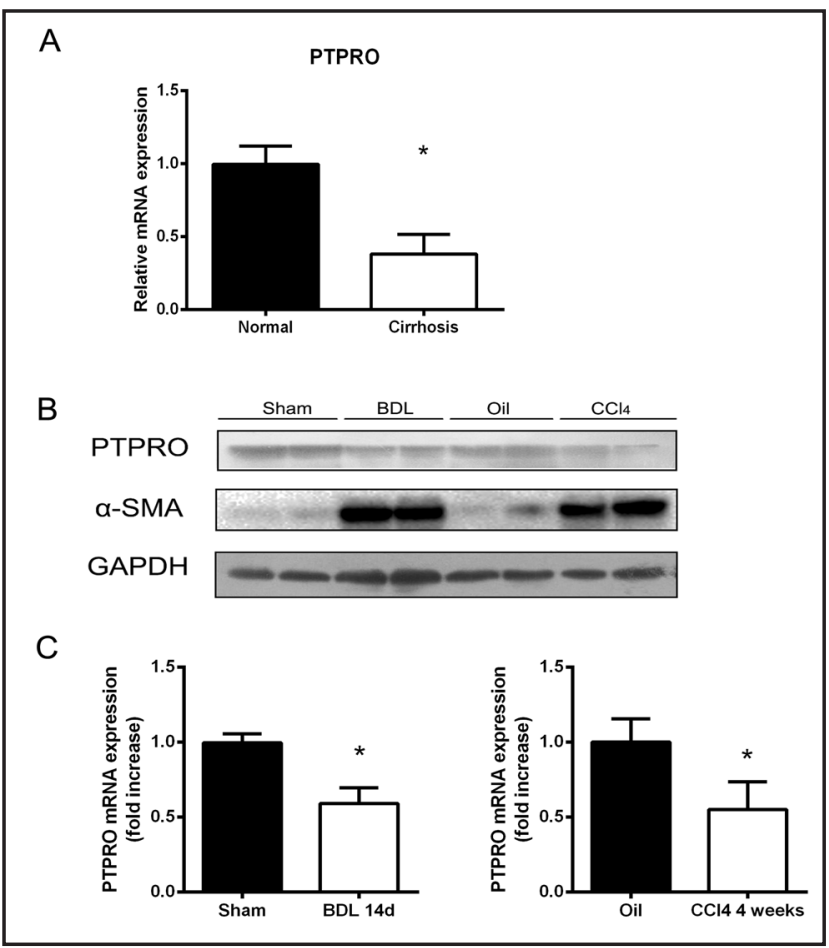

Second, to ascertain if HSC activation was also associated with enhanced hepatic fibrogenesis, mRNA for profibrogenic markers, such as collagen type $1 \alpha 1$ (Col1 $\alpha 1$ ) was significantly lower in BDL- or $\mathrm{CCl}_{4}$-challenged $\mathrm{PTPRO}^{-/-}$mice. Tissue inhibitor of metalloproteinase (TIMP)-1, matrix metalloproteinase (MMP)-2 and another important fibrosis- related factor, transforming growth factor (TGF)- $\beta 1$, also exhibited the same trend (Fig. 3B and 3C). These experiments confirmed that PTPRO plays an important role in BDLand $\mathrm{CCl}_{4}$-induced liver fibrosis, and PTPRO deficiency may decrease the degree of liver fibrosis.

PTPRO is downregulated in mouse and human fibrotic liver

Since PTPRO deficiency largely protect the mice from liver, we collected cirrhotic $(n=18)$ and normal $(n=8)$ liver tissue for real-time PCR for clinical verification. We found that PTPRO mRNA in patient samples was strongly downregulated in comparison with normal liver $(P<0.05$; Fig. 4A). To determine whether PTPRO played a critical role in liver fibrogenesis in vivo, we induced two different experimental fibrogenesis models by BDL and $\mathrm{CCl}_{4}$ administration in wild-type (WT) mice. $\alpha$-SMA, a cardinal marker for HSC activation, was upregulated in fibrosis specimens, indicating that fibrosis was induced in both models. We found marked reduction of PTPRO expression in fibrotic liver at the mRNA and protein levels, suggesting that PTPRO participates in pathological progress (Fig. 4B, 4C).

\section{Effect of PTPRO in HSC and hepatocyte activation and downstream signaling}

Previous research has shown that PTPRO is significantly downregulated in clinical fibrotic liver specimens and experimental fibrotic mouse liver, while PTPRO KO largely protects mice from BDL- and $\mathrm{CCl}_{4}$-induced chronic liver injury and fibrogenesis. To address this paradox, we clarified the role of PTPRO in liver fibrosis at the cellular level.

Transactivation of quiescent HSCs is thought to be a key step in collagen deposition and ECM accumulation in liver cirrhosis [3]. During HSC activation, numerous pathways are activated as paracrine or autocrine signaling. PDGF is the classic cytokine which could be released during hepatocellular damage and trigger downstream signaling by PI3K/AKT, ERK and other pathways $[25,26]$. A recent study found that PDGF is a vital direct mitogen and information regulator for hepatocytes $[27,28]$. We first validated that alteration of 


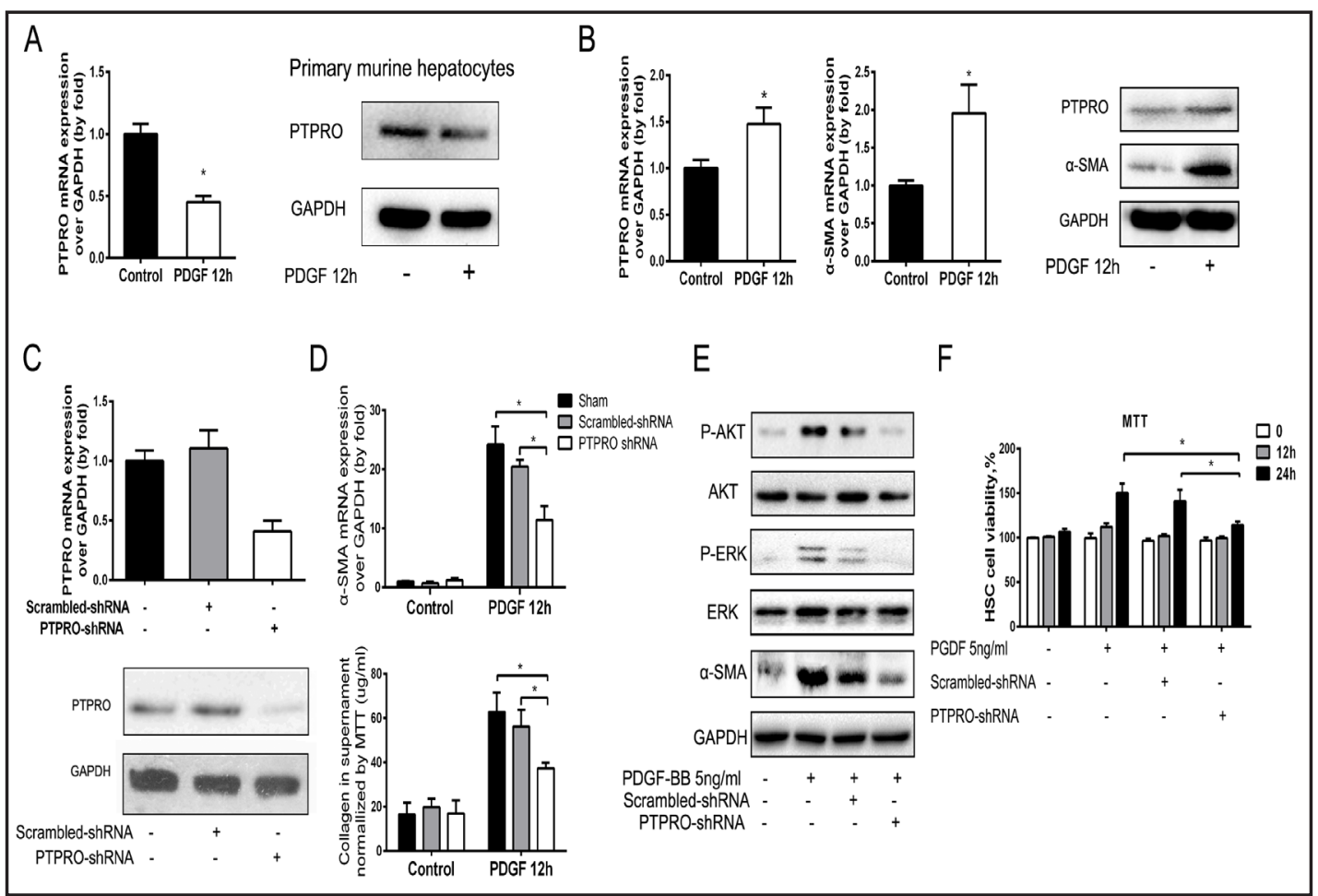

Fig. 5. Effect of PTPRO in HSC activation and downstream signaling. Mouse HSCs were isolated from naïve C57BL/ 6 mice and stimulated as previously described. (A) RNA and protein of PTPRO in isolated hepatocytes after PDGF-BB administration for $12 \mathrm{~h}$. (B) After $12 \mathrm{~h}$ restimulation, PDGF-treated HSCs were analyzed for PTPRO and $\alpha$-SMA at the RNA and protein levels, compared with original HSCs. (C) Twenty-four hours PTPRO-shRNA transfection resulted in a significant decrease in PTPRO expression in comparison with the same cells transfected with scrambled-shRNA, by quantitative real-time PCR and western blotting. (D) Under rmPDFG-BB (5 ng/ml) treatment for $12 \mathrm{~h}$, $\alpha$-SMA transcription in primary HSCs was attenuated by PTPRO-shRNA transfection. The same trend was observed for soluble collagen assessed by Sircol assay. The collagen concentration was standardized by MTT viability. (E) PDGF-BB administration activated HSCs through intracellular AKT and ERK phosphorylation, while PTPRO-shRNA significantly reduced kinase activation and subsequently attenuated $\alpha$-SMA production. (F) PTPRO-shRNA decreased HSC proliferation at 12 and $24 \mathrm{~h}$ after rmPDGF-BB treatment, as compared to the scrambled-siRNA transfected and naive cells $\left({ }^{*} P<0.05\right)$.

PTPRO gene expression during $12 \mathrm{~h}$ stimulation of primary hepatocytes and HSCs with nonlethal concentrations of PDGF-BB. PTPRO transcription was significantly decreased in hepatocytes during PDGF-BB administration, while the opposite effect was seen in HSCs (Figure 5A and 5B).

To establish the critical role of PTPRO in activated HSCs, shRNA specific for mouse PTPRO (PTPRO-shRNA) was used to knockdown PTPRO expression. As illustrated in Fig. 5C, PTPROshRNA induced a significant decrease in PTPRO levels in HSCs after $24 \mathrm{~h}$ transfection, in comparison to the cells transfected with scrambled shRNA (Fig. 5C). Moreover, we observed a strong increase of $\alpha$-SMA transcription and collagen release in PDGF-BB-treated primary HSCs and supernatant, respectively, which indicated that PDGF-BB-activated HSCs could be the main sources of ECM and collagen. PTPRO knockdown also reduced HSC activation by controlling expression of $\alpha$-SMA and soluble collagen (Fig. 5D).

Next, we determined if silencing PTPRO affected the activity of PI3K and ERK by analyzing the protein levels of p-AKT and p-ERK in HSCs after PTPRO downregulation. Western blotting showed a decrease in both p-AKT and p-ERK in activated HSCs transfected with PTPRO-shRNA in comparison to the scrambled-shRNA-treated cells after PDGF-BB 
restimulation (Fig. 5E). These data suggest that PTPRO affects HSC activation through p-AKT and p-ERK signaling pathways, which may be attributed to the PI3K pathway [29]. The viability of HSCs after $24 \mathrm{~h}$ incubation with PDGF-BB and PTPRO-shRNA decreased in the MTT assay, as compared to cell treated with PDGF-BB and transfected with scrambled siRNA (Fig. 5F). This indicated that knockdown of PTPRO could neutralize PDGFBB-induced HSC proliferation, while no significant differences were observed after $12 \mathrm{~h}$ incubation.

\section{Discussion}

Although liver fibrosis is a critical stage in the mortality of chronic liver diseases, the pathological mediator for fibrogenesis has not been fully clarified [30]. A key event leading to liver damage is the transition of quiescent HSCs into activated myofibroblasts [31]. Therefore, inhibition of HSC activation/proliferation results primarily from recruitment of inflammatory cells or subsequent production of cytokines, and blockade of ECM formation is a key strategy for therapeutic intervention.

PTPRO is an important member of the receptor-type PTP family, which is implicated in several biological processes. Low or aberrant expression of PTPRO has been shown in many types of human cancer, including lung cancer, hepatocellular carcinoma, and breast cancer $[18,32,33]$. Research also shows that PTPRO inhibitors can significantly reduce the pathophysiological severity in a mouse model of allergic dermatitis and inflammatory bowel disease [34].. Other PTPs play a regulatory role in fibrosis. For instance, PTP- $\alpha$ promotes profibrotic signaling pathways in lung fibroblasts through control of cellular responsiveness to TGF- $\beta$ [35]. Orthovanadate as a PTP inhibitor suppresses HSC activation and liver fibrosis in rats [36]. PTEN (phosphatase and tension homolog) is a critical tumor suppressor in various types of cancer, whose reduction is followed by activation of the PI3K/ AKT and ERK pathways, resulting in HSC activation [37]. How PTPRO acts in liver fibrosis is still unknown. In our current study, we utilized gene-deficient mice and demonstrated that PTPRO expression was reduced in mouse and human fibrotic liver tissue, and that PTPRO KO or knock-down plays a critical role in emolliating collagen deposition and HSC activation via PDGF/AKT and ERK pathways.

We demonstrated mice deficient in PTPRO displayed a significant reduction in hepatic inflammation and fibrogenesis in two different models. At the meantime, the expression of PTPRO in both human cirrhotic tissue and mouse fibrosis models are downregulated. So, hepatocytes and HSCs were investigated separately in vitro. We found that PDGF-BB stimulation reduced PTPRO expression in hepatocytes but increased its production in HSCs. Considering that hepatocytes comprise the majority of hepatic cells, downregulated PTPRO in whole liver homogenate might not reflect the actual situation in different cell types. Although the mechanism underlying this phenomenon is still unclear, we further demonstrated that inhibition of PTPRO had profound antifibrogenic effects and improved survival in BDL mice.

PTPRO plays a critical role in aberrant carcinogenesis, therefore, we tried to verify the exact consequences in BDL- and $\mathrm{CCl}_{4}$-induced chronic liver injury and fibrosis. BDL operation and $\mathrm{CCl}_{4}$ injection contained autoimmune activation and acute inflammation stimulate, which are the well simulate for human primary biliary cirrhosis and hepatitis B virus infection associated liver fibrosis [38, 39]. We found that PTPRO deficiency largely protected mice from repeated challenges that cause liver injury, profibrotic cytokine expression, and fibrosis with collagen deposition. TGF- $\beta$ as the classical profibrotic factor enhancing collagen and ECM deposition through the Smad2 and Smad3 pathway is PTPRO dependent [40]. Although further investigation on the relation between TGF- $\beta$ and PTPRO is lacking, PTPRO KO is assumed to have a protective effect in liver cirrhosis.

PDGF is a potent inducer of HSC and hepatocyte activation and proliferation [27, 41]. It has been shown that both the ERK and PI3K/AKT pathways are markedly activated following stimulation with PDGF and other growth factors and subsequently promote HSC activation 
$[29,42]$. Mouse models simulate a complex pathophysiological syndrome including chronic inflammation injury and the multilineage antimitochondrial response that causes human cirrhosis $[38,43]$. Considering that viability of HSCs isolated from PTPRO $^{-/-}$mice is not satisfied for the following test and PTPRO deletion is not liver specific, which could affect experiment result, we utilized HSCs isolated from livers of C57BL/6 mice following shRNA treatment to minimize the above influence. We found that PTPRO expression was enhanced by PDGF, and knockdown of PTPRO with shRNA strongly inhibited PDGF-induced proliferation and $\alpha$-SMA expression, which suppressed the phosphorylation of PDGF/AKT and PDGF/ERK simultaneously in activated HSCs. PTPRO expression was notably reduced in the primary murine hepatocytes after treatment with PDGF for $12 \mathrm{~h}$. However hepatic fibrogenesis is a complex response mediated by many different cell populations, with ECM accumulation representing the final step. It is conceivable that other cells such as Kupffer cells and hepatocytes are also involved during the wound-healing response [26]. PTPRO plays a dual role in hepatic I/R injury, and can act as a marker of acute podocyte injury in rat nephrosis [22]. PTPRO expression attenuates STAT3 activity, which in hepatocytes plays a protective role in preventing liver fibrosis [44,45]. Research has also found that upregulation of STAT3 phosphorylation is dependent on Kupffer-cell-derived IL-6 secretion [46]. PTPRO expression in vivo may also be an indirect cooperating with a direct mechanism in HSC activation and progression of liver fibrosis. The level of PTPRO expression could also be multi-azimuth controlled by HSCs, Kupffer cells and hepatocytes in vivo, which could account for the different trends between PDGF-induced HSC activation in vitro and liver homogenate of human and mouse model tissues. Interestingly, we have recently shown that PTPRO expression inhibits progression of hepatocellular carcinoma through downregulating STAT3 [21]. Since liver fibrosis are the precancerous lesion of hepatocellular carcinoma, PTPRO may served as an pro-fibrotic and an anti-oncogenic role at the same time. We speculate that PTPRO may play a different role depending on the cell type, for instance hepatocytes or HSC. As we shown, PTPRO dependent HSC activation contributes to the ECM and collagen deposition but PTPRO attenuated STAT3 activation inhibits HCC progression. On the other hand, the role of PTPRO in immune regulation and surveillance are also important in HCC development which need more meticulous research.

As research continues, liver fibrosis is associated with a growing number of factors including hypoxia and immunoregulation $[47,48]$. PDGF, an important signaling pathways involved in hypoxia associated liver fibrosis and HSC activation and proliferation [49], which are currently developed as new antifibrotic drugs target, and have many breakthroughs, including brivanib and nilotinib [50,51]. Sodium orthovanadate is a PTP inhibitor and a pivotal regulator of profibrotic mechanisms that affect the PDGF pathway [36, 52]. This study shows the positive role of PTPRO in liver fibrosis, which makes a major contribution to understanding the pharmacology of these drugs and drives the discovery of new drugs.

Although the findings suggested a conviced role of PTPRO in regulation of liver fibrosis, more work is necessary to clarify the detail of the signaling pathway and translate this into therapy that alleviates the burden and susceptibility of these disorders in humans.

\section{Disclosure Statement}

All authors have contributed substantially to this work and declare no conflict of interest.

\section{Acknowledgments}

This work was supported by grants from the National Natural Science Foundation for Distinguished Young Scholars (81225017 to B.S.), National Basic Research Program of China (2012CB910800 to B.S.), National Natural Science Foundation (81430062 to B.S.; 81302106 to J.H.; 81201528 to R.J.). This work also supported in part by the program for 


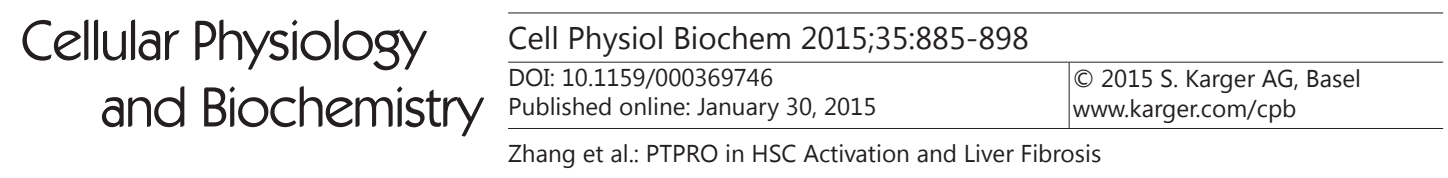

Development of Innovative Research Team in the First Affiliated Hospital of NJMU, and the Priority Academic Program of Jiangsu Higher Education Institutions. B.S. is Yangtze River scholars Distinguished Professor.

\section{Reference}

1 Bataller R, Brenner DA: Liver fibrosis. J Clin Invest 2005;115:209-218.

-2 Cassiman D, Libbrecht L, Desmet V, Denef C, Roskams T: Hepatic stellate cell/myofibroblast subpopulations in fibrotic human and rat livers. J Hepatol 2002;36:200-209.

-3 Friedman SL: Hepatic stellate cells: Protean, multifunctional, and enigmatic cells of the liver. Physiol Rev 2008;88:125-172.

4 Lee UE, Friedman SL: Mechanisms of hepatic fibrogenesis. Best Pract Res Clin Gastroenterol 2011;25:195206.

5 Friedman SL: Evolving challenges in hepatic fibrosis. Nature RevGastroenterol Hepatol 2010;7:425-436.

6 Leask A, Abraham DJ: Tgf-beta signaling and the fibrotic response. FASEB J 2004;18:816-827.

7 Czochra P, Klopcic B, Meyer E, Herkel J, Garcia-Lazaro JF, Thieringer F, Schirmacher P, Biesterfeld S, Galle PR, Lohse AW, Kanzler S: Liver fibrosis induced by hepatic overexpression of pdgf-b in transgenic mice. J Hepatol 2006;45:419-428.

8 Leclercq IA, Farrell GC, Schriemer R, Robertson GR: Leptin is essential for the hepatic fibrogenic response to chronic liver injury. J Hepatol 2002;37:206-213.

-9 Meng F, Wang K, Aoyama T, Grivennikov SI, Paik Y, Scholten D, Cong M, Iwaisako K, Liu X, Zhang M, Osterreicher CH, Stickel F, Ley K, Brenner DA, Kisseleva T: Interleukin-17 signaling in inflammatory, kupffer cells, and hepatic stellate cells exacerbates liver fibrosis in mice. Gastroenterology 2012;143:765-776 e761-763.

10 Tan Z, Qian X, Jiang R, Liu Q, Wang Y, Chen C, Wang X, Ryffel B, Sun B: Il-17a plays a critical role in the pathogenesis of liver fibrosis through hepatic stellate cell activation. J Immunol 2013;191:1835-1844.

11 McHedlidze T, Waldner M, Zopf S, Walker J, Rankin AL, Schuchmann M, Voehringer D, McKenzie AN, Neurath MF, Pflanz S, Wirtz S: Interleukin-33-dependent innate lymphoid cells mediate hepatic fibrosis. Immunity 2013;39:357-371.

12 Tonks NK: Protein tyrosine phosphatases: From genes, to function, to disease. Nat Rev Mol Cell Biol 2006; 7:833-846.

13 Matozaki T, Murata Y, Mori M, Kotani T, Okazawa H, Ohnishi H: Expression, localization, and biological function of the $\mathrm{r} 3$ subtype of receptor-type protein tyrosine phosphatases in mammals. Cell Signal 2010;22:1811-1817.

14 Chen B, Bixby JL: A novel substrate of receptor tyrosine phosphatase ptpro is required for nerve growth factor-induced process outgrowth. J Neurosci 2005;25:880-888.

15 Liao WH, Cheng CH, Hung KS, Chiu WT, Chen GD, Hwang PP, Hwang SP, Kuan YS, Huang CJ: Protein tyrosine phosphatase receptor type o (ptpro) regulates cerebellar formation during zebrafish development through modulating fgf signaling. Cell Mol Life Sci 2013;70:2367-2381.

-16 Yu M, Lin G, Arshadi N, Kalatskaya I, Xue B, Haider S, Nguyen F, Boutros PC, Elson A, Muthuswamy LB, Tonks NK, Muthuswamy SK: Expression profiling during mammary epithelial cell three-dimensional morphogenesis identifies ptpro as a novel regulator of morphogenesis and erbb2-mediated transformation. Mol Cell Biol 2012;32:3913-3924.

17 You YJ, Chen YP, Zheng XX, Meltzer SJ, Zhang H: Aberrant methylation of the ptpro gene in peripheral blood as a potential biomarker in esophageal squamous cell carcinoma patients. Cancer Lett 2012;315:138-144.

18 Motiwala T, Kutay H, Ghoshal K, Bai S, Seimiya H, Tsuruo T, Suster S, Morrison C, Jacob ST: Protein tyrosine phosphatase receptor-type o (ptpro) exhibits characteristics of a candidate tumor suppressor in human lung cancer. Proc Natl Acad Sci U S A 2004;101:13844-13849.

19 Motiwala T, Ghoshal K, Das A, Majumder S, Weichenhan D, Wu YZ, Holman K, James SJ, Jacob ST, Plass C: Suppression of the protein tyrosine phosphatase receptor type o gene (ptpro) by methylation in hepatocellular carcinomas. Oncogene 2003;22:6319-6331. 


\section{Cellular Physiology Cell Physiol Biochem 2015;35:885-898 and Biochemistry

20 Ramaswamy B, Majumder S, Roy S, Ghoshal K, Kutay H, Datta J, Younes M, Shapiro CL, Motiwala T, Jacob ST: Estrogen-mediated suppression of the gene encoding protein tyrosine phosphatase ptpro in human breast cancer: Mechanism and role in tamoxifen sensitivity. Mol Endocrinol 2009;23:176-187.

21 Hou J, Xu J, Jiang R, Wang Y, Chen C, Deng L, Huang X, Wang X, Sun B: Estrogen-sensitive ptpro expression represses hepatocellular carcinoma progression by control of stat3. Hepatology 2013;57:678-688.

22 Hou J, Xia Y, Jiang R, Chen D, Xu J, Deng L, Huang X, Wang X, Sun B: Ptpro plays a dual role in hepatic ischemia reperfusion injury through feedback activation of nf-kappab. J Hepatol 2014;60:306-312.

-23 Fan X, Zhang Q Li S, Lv Y, Su H, Jiang H, Hao Z: Attenuation of ccl4-induced hepatic fibrosis in mice by vaccinating against tgf-beta1. PLoS One 2013;8:e82190.

24 Seki E, De Minicis S, Gwak GY, Kluwe J, Inokuchi S, Bursill CA, Llovet JM, Brenner DA, Schwabe RF: Ccr1 and ccr5 promote hepatic fibrosis in mice. J Clin Invest 2009;119:1858-1870.

-25 Lechuga CG, Hernandez-Nazara ZH, Hernandez E, Bustamante M, Desierto G, Cotty A, Dharker N, Choe M, Rojkind M: Pi3k is involved in pdgf-beta receptor upregulation post-pdgf-bb treatment in mouse hsc. Am J Physiol Gastrointest Liver Physiol 2006;291:G1051-1061.

-26 Friedman SL: Mechanisms of hepatic fibrogenesis. Gastroenterology 2008;134:1655-1669.

-27 Sonnweber T, Nachbaur D, Schroll A, Nairz M, Seifert M, Demetz E, Haschka D, Mitterstiller AM, Kleinsasser A, Burtscher M, Trubsbach S, Murphy AT, Wroblewski V, Witcher DR, Mleczko-Sanecka K, Vecchi C, Muckenthaler MU, Pietrangelo A, Theurl I, Weiss G: Hypoxia induced downregulation of hepcidin is mediated by platelet derived growth factor bb. Gut 2014;63:1951-1959.

28 Bowen WC, Michalopoulos AW, Orr A, Ding MQ Stolz DB, Michalopoulos GK: Development of a chemically defined medium and discovery of new mitogenic growth factors for mouse hepatocytes: Mitogenic effects of fgf1/2 and pdgf. PLoS One 2014;9:e95487.

29 Fang L, Zhan S, Huang C, Cheng X, Lv X, Si H, Li J: Trpm7 channel regulates pdgf-bb-induced proliferation of hepatic stellate cells via pi3k and erk pathways. Toxicol Appl Pharmacol 2013;272:713-725.

-30 Friedman SL: Liver fibrosis -- from bench to bedside. J Hepatol 2003;38:S38-53.

-31 Lisbonne M, L'Helgoualc'h A, Nauwelaers G, Turlin B, Lucas C, Herbelin A, Piquet-Pellorce C, Samson M: Invariant natural killer t-cell-deficient mice display increased ccl(4) -induced hepatitis associated with cxcl1 over-expression and neutrophil infiltration. Eur J Immunol 2011;41:1720-1732.

-32 Xu X, Hong Y, Kong C, Xu L, Tan J, Liang Q, Huang B, Lu J: Protein tyrosine phosphatase receptor-type o (ptpro) is co-regulated by e2f1 and mir-17-92. FEBS Lett 2008;582:2850-2856.

33 Pierga JY, Petit T, Delozier T, Ferrero JM, Campone M, Gligorov J, Lerebours F, Roche H, Bachelot T, CharafeJauffret E, Pavlyuk M, Kraemer S, Bidard FC, Viens P: Neoadjuvant bevacizumab, trastuzumab, and chemotherapy for primary inflammatory her2-positive breast cancer (beverly-2): An open-label, singlearm phase 2 study. Lancet Oncol 2012;13:375-384.

34 Gobert RP, van den Eijnden M, Szyndralewiez C, Jorand-Lebrun C, Swinnen D, Chen L, Gillieron C, Pixley F, Juillard P, Gerber P, Johnson-Leger C, Halazy S, Camps M, Bombrun A, Shipp M, Vitte PA, Ardissone V, Ferrandi C, Perrin D, Rommel C, Hooft van Huijsduijnen R: Glepp1/protein-tyrosine phosphatase phi inhibitors block chemotaxis in vitro and in vivo and improve murine ulcerative colitis. J Biol Chem 2009;284:11385-11395.

-35 Aschner Y, Khalifah AP, Briones N, Yamashita C, Dolgonos L, Young SK, Campbell MN, Riches DW, Redente EF, Janssen WJ, Henson PM, Sap J, Vacaresse N, Kapus A, McCulloch CA, Zemans RL, Downey GP: Protein tyrosine phosphatase alpha mediates profibrotic signaling in lung fibroblasts through tgf-beta responsiveness. Am J Pathol 2014;184:1489-1502.

-36 Nishikawa Y, Ohi N, Yagisawa A, Doi Y, Yamamoto Y, Yoshida M, Tokairin T, Yoshioka T, Omori Y, Enomoto K: Suppressive effect of orthovanadate on hepatic stellate cell activation and liver fibrosis in rats. Am J Pathol 2009;174:881-890.

37 Bian EB, Huang C, Ma TT, Tao H, Zhang H, Cheng C, Lv XW, Li J: Dnmt1-mediated pten hypermethylation confers hepatic stellate cell activation and liver fibrogenesis in rats. Toxicol Appl Pharmacol 2012;264:1322.

-38 Hirschfield GM, Gershwin ME: The immunobiology and pathophysiology of primary biliary cirrhosis. Annu Rev Pathol 2013;8:303-330.

-39 Bataller R, Gabele E, Parsons CJ, Morris T, Yang L, Schoonhoven R, Brenner DA, Rippe RA: Systemic infusion of angiotensin ii exacerbates liver fibrosis in bile duct-ligated rats. Hepatology 2005;41:1046-1055. 


\section{Cellular Physiology $\quad$ Cell Physiol Biochem 2015;35:885-898 and Biochemistry \\ Zhang et al.: PTPRO in HSC Activation and Liver Fibrosis}

40 Liu C, Gaca MD, Swenson ES, Vellucci VF, Reiss M, Wells RG: Smads 2 and 3 are differentially activated by transforming growth factor-beta (tgf-beta ) in quiescent and activated hepatic stellate cells. Constitutive nuclear localization of smads in activated cells is tgf-beta-independent. J Biol Chem 2003;278:1172111728.

41 Melton AC, Yee HF: Hepatic stellate cell protrusions couple platelet-derived growth factor-bb to chemotaxis. Hepatology 2007;45:1446-1453.

42 Marra F, Arrighi MC, Fazi M, Caligiuri A, Pinzani M, Romanelli RG, Efsen E, Laffi G, Gentilini P: Extracellular signal-regulated kinase activation differentially regulates platelet-derived growth factor's actions in hepatic stellate cells, and is induced by in vivo liver injury in the rat. Hepatology 1999;30:951-958.

43 Kahraman A, Barreyro FJ, Bronk SF, Werneburg NW, Mott JL, Akazawa Y, Masuoka HC, Howe CL, Gores GJ: Trail mediates liver injury by the innate immune system in the bile duct-ligated mouse. Hepatology 2008;47:1317-1330.

44 Mair M, Zollner G, Schneller D, Musteanu M, Fickert P, Gumhold J, Schuster C, Fuchsbichler A, Bilban M, Tauber S, Esterbauer H, Kenner L, Poli V, Blaas L, Kornfeld JW, Casanova E, Mikulits W, Trauner M, Eferl R: Signal transducer and activator of transcription 3 protects from liver injury and fibrosis in a mouse model of sclerosing cholangitis. Gastroenterology 2010;138:2499-2508.

45 Horiguchi N, Lafdil F, Miller AM, Park O, Wang H, Rajesh M, Mukhopadhyay P, Fu XY, Pacher P, Gao B: Dissociation between liver inflammation and hepatocellular damage induced by carbon tetrachloride in myeloid cell-specific signal transducer and activator of transcription 3 gene knockout mice. Hepatology 2010;51:1724-1734.

46 Deng YR, Ma HD, Tsuneyama K, Yang W, Wang YH, Lu FT, Liu CH, Liu P, He XS, Diehl AM, Gershwin ME, Lian ZX: Stat3-mediated attenuation of ccl4-induced mouse liver fibrosis by the protein kinase inhibitor sorafenib. J Autoimmun 2013;46:25-34.

$\$ 47$ Troeger JS, Schwabe RF: Hypoxia and hypoxia-inducible factor 1alpha: Potential links between angiogenesis and fibrogenesis in hepatic stellate cells. Liver Int 2011;31:143-145.

-48 Paik YH, Schwabe RF, Bataller R, Russo MP, Jobin C, Brenner DA: Toll-like receptor 4 mediates inflammatory signaling by bacterial lipopolysaccharide in human hepatic stellate cells. Hepatology 2003;37:1043-1055.

49 Pinzani M: Pdgf and signal transduction in hepatic stellate cells. Front Biosci 2002;7:d1720-1726.

-50 Nakamura I, Zakharia K, Banini BA, Mikhail DS, Kim TH, Yang JD, Moser CD, Shaleh HM, Thornburgh SR, Walters I, Roberts LR: Brivanib attenuates hepatic fibrosis in vivo and stellate cell activation in vitro by inhibition of fgf, vegf and pdgf signaling. PLoS One 2014;9:e92273.

51 Liu Y, Wang Z, Kwong SQ Lui EL, Friedman SL, Li FR, Lam RW, Zhang GC, Zhang H, Ye T: Inhibition of pdgf, tgf-beta, and abl signaling and reduction of liver fibrosis by the small molecule bcr-abl tyrosine kinase antagonist nilotinib. J Hepatol 2011;55:612-625.

-52 Klironomos S, Notas G, Sfakianaki O, Kiagiadaki F, Xidakis C, Kouroumalis E: Octreotide modulates the effects on fibrosis of tnf-alpha, tgf-beta and pdgf in activated rat hepatic stellate cells. Regul Pept 2014;188:5-12. 